\title{
Measurement and simulation of neutron monitors count rate dependence on surrounding structure
}

\author{
N. Aiemsa-ad,,$^{a, b, c}$ D. Ruffolo, ${ }^{c, d}$ A. Sáiz, ${ }^{c, d}$ P.-S. Mangeard ${ }^{*}, e$ T. Nutaro, ${ }^{c, f}$ W. \\ Nuntiyakul, ${ }^{c, d, g}$ N. Kamyan, ${ }^{d}$ T. Khumlumlert, ${ }^{a}$ H. Krüger, ${ }^{h}$ H. Moraal, ${ }^{h}$ J. W. Bieber, ${ }^{i}$ \\ J. Clem, ${ }^{i}$ P. Evenson ${ }^{i}$
}

${ }^{a}$ Naresuan University, Phitsanulok, Thailand. ${ }^{b}$ Rajabhat Rajanagarindra University, Chachoengsao, Thailand. ${ }^{c}$ Thailand Center of Excellence in Physics, CHE, Ministry of Education, Bangkok, Thailand. ${ }^{d}$ Mahidol University, Bangkok, Thailand. ${ }^{e}$ National Astronomical Research Institute of Thailand (NARIT), Chiang Mai, Thailand. ${ }^{f}$ Ubon Ratchathani University, Ubon Ratchathani, Thailand. ${ }^{g}$ Chandrakasem Rajabhat University, Bangkok,

Thailand. ${ }^{h}$ North-West University, Potchefstroom, South Africa. ${ }^{i}$ Bartol Research Institute, University of Delaware, Newark, USA.

E-mail: nalinee_nook@hotmail.com, david.rufemahidol.ac.th, alejandro.sai@mahidol.ac.th, psmangeardegmail.com,

tnutaro@yahoo.com, w. nuntiyakul@gmail.com, p_chang24@hotmail.com, thiraneek@nu.ac.th, helena.kruger@nwu.ac.za, harm.moraal@nwu.ac.za, jwbieber@bartol. udel. edu, clemebartol . udel . edu,

evenson@bartol.udel.edu

Neutron monitors are the premier instruments for precise measurements of time variations (e.g., of solar origin) in the Galactic cosmic ray (GCR) flux in the range of $\sim 1-100 \mathrm{GeV}$. However, it has proven challenging to accurately determine the yield function (efficiency) vs. rigidity in order to relate a neutron monitor's count rate with those of other monitors in the worldwide network and the underlying GCR spectrum. Monte Carlo simulations of the yield function have been developed but there have been few opportunities to validate these models observationally, especially with regard to the particular environment surrounding each monitor. Here we have precisely measured the count rate of a calibration neutron monitor ("calibrator") near the Princess Sirindhorn Neutron Monitor (PSNM) at Doi Inthanon, Thailand, which provides a basis for comparison with count rates of other neutron monitors worldwide that are similarly calibrated. We directly measured the effect of the surrounding structure by operating the calibrator both outside and inside the building. Using Monte Carlo simulations, we can clarify differences in response of the calibrator and PSNM, as well as the calibrator outside and inside the building. The dependence of the calibrator count rate on surrounding structure can be attributed to its sensitivity to neutrons of 0.5-10 MeV and a shift of sensitivity to nucleons of higher energy when placed inside the building. Simulation results for the calibrator to PSNM count rate ratio are in agreement to within a few percent, providing a useful validation and improving confidence in our ability to model the yield function for a neutron monitor station.

The 34th International Cosmic Ray Conference,

30 July- 6 August, 2015

The Hague, The Netherlands 


\section{Introduction}

Neutron Monitors (NMs) are ground-based detectors that count the secondary particles (SPs, mostly neutrons) produced by the interaction of the cosmic primary particles (PPs) in the atmosphere. NMs provide a unique measurement of the time variations of the Galactic cosmic rays (GCRs) that are mostly induced by the 11-22 year solar cycles, the Sun's 27-day synodic period as well as by shorter term variations such as solar storms. Independently of its technical characteristics, the rigidity range of the sensitivity of a NM to the fluctuations of the GCR spectrum depends on its location and on its altitude. Indeed, the strength of the geomagnetic field at the location of the detector, usually characterized by its vertical cutoff rigidity $P_{c}$ (from 1 to $17 \mathrm{GV}$ ), prevents the PPs with a rigidity lower than the cutoff from reaching Earth's atmosphere and producing an atmospheric shower with SPs reaching the detector. Multiple NM combined analyses could in principle improve our understanding of the GCR flux variations via the study of their anisotropy with a good time scale precision (1 hour). An inter-calibration at the level of $0.2 \%$ [15] between the NMs of the worldwide network is required to combine their count rates. Achieving such level of accuracy passes by significant improvements towards the determination of the effective area, also called the yield function, of a NM. Recent works showed encouraging progress by fitting data (from spacecraft and latitude surveys) [3] and from Monte Carlo (MC) simulation [5, 13]. Further effort is still needed to understand the remaining discrepancies between MC hadron interaction generators and the implementation of the heavy nuclei contribution [14]. Moreover, the yield function depends on the intrinsic characteristics of each NM, e.g., the electronic dead time, the surroundings and the atmospheric profile at the location of the detector.

We present here the results from the operations of a portable calibration neutron monitor (hereafter, the "calibrator") [10] in conjunction with the Princess Sirindhorn Neutron Monitor (PSNM) at the summit of Doi Inthanon, Thailand $\left(18.59^{\circ} \mathrm{N}, 98.49^{\circ} \mathrm{E}, 2565 \mathrm{~m}\right)$ with the world's highest vertical cutoff rigidity $P_{c}=16.8 \mathrm{GV}$. We report here the count rate of PSNM with respect to the calibrator count rate and thus to other stations, including SANAE, Potchestroom and Kiel $[11,16]$ as well as to the future calibrated NM. The effects of the surroundings were also studied by operating the calibrator inside and outside the building. That was the opportunity to validate our computation of the atmospheric shower over Doi Inthanon and our model of the interactions of the SPs in the PSNM station.

\section{The experiment}

A detailed description of the equipment and observations is available in [1]. Briefly, the calibrator $[9,10]$ is relatively compact $(753 \mathrm{~mm}$ long and $223 \mathrm{~kg}$ ) allowing it to be transported to the location of the NM that will be calibrated. The neutrons are detected with a proportional counter via the reaction ${ }^{3} \mathrm{He}(n, p)^{3} \mathrm{H}$. It was deployed at the location of the PSNM, a 18-tube NM64 neutron monitor [4] that measures the neutrons via the reaction ${ }^{10} \mathrm{~B}(n, \alpha){ }^{7} \mathrm{Li}$. The count rate of the calibrator is $\sim 200$ times smaller than in the 18NM64. We focus here on the relative count rate of the calibrator with respect to that of PSNM, i.e., the Cal/NM ratio, for two different experimental setups. First, the calibrator operated outside the station from February 11 to April 6, 2010 with a relatively

\footnotetext{
* Speaker.
} 


\begin{tabular}{lccc}
\hline & \multicolumn{2}{c}{ Calibrator } & PSNM \\
\cline { 2 - 3 } & Outside & Inside & \\
\hline Observations: & & & \\
Count rate for calibrator outside & $10,674(6)$ & - & $2.1964(9) \times 10^{6}$ \\
Count rate for calibrator inside & - & $11,160(7)$ & $2.2289(10) \times 10^{6}$ \\
Count rate, relative to PSNM & $4.860(2) \times 10^{-3}$ & $5.007(2) \times 10^{-3}$ & 1 \\
\hline Monte Carlo: & & & \\
Count rate & $12,148(75)$ & $13,126(143)$ & $2.502(2) \times 10^{6}$ \\
Count rate, relative to PSNM & $4.86(3) \times 10^{-3}$ & $5.25(6) \times 10^{-3}$ & 1 \\
\hline
\end{tabular}

Table 1: Observational and simulation results for calibrator outside and inside the building. Parentheses indicate statistical standard error in the final digit(s). All count rates are hourly uncorrected rates [1].

standard configuration with respect to prior calibrations of NMs [11]. It consists of operating the calibrator $5 \mathrm{~cm}$ above a pool of water $(65 \mathrm{~cm}$ height) in order to moderate the neutrons produced at the surface underneath, in open atmosphere and away from any construction that could disturb the spectrum of the secondary particles. In our case, the calibrator was installed on a bunker several meters from the station as shown in Figure 1. The second setup consists of operating the calibrator within the PSNM station (Figure 2), without the pool, and was performed from June 11 to June 28, 2010. Note here that those periods corresponded to low solar activity. During both configurations, the 18NM64 and the calibrator were equipped with the same electronic boards developed by the Bartol Research Institute (BRI), Delaware, USA. Originally designed for indoor only use, the BRI electronics presented some noise during the outdoor operations with a temperature $\mathrm{T}>25^{\circ} \mathrm{C}$. The standard hourly count rate (CT) of the calibrator was consequently not used and was replaced by the pulse height $(\mathrm{PH})$ count rate calculated through an alternative circuit. Despite better robustness, the $\mathrm{PH}$ data were also affected by the difficult meteorological conditions with temperature in the electronics head rising up to $60{ }^{\circ} \mathrm{C}$ in the daytime. The noisy hours were removed from the analysis. The indoor operations were not affected by this problem. Otherwise no significant correlation was observed between the Cal/NM ratio and the counter temperature. The observed hourly count rates are presented in the first two rows of Table 1 and the corresponding Cal/NM ratios in the third row.

It is well known that the NM count rate depends on the in-situ atmospheric pressure, $p$. The common correction depends on two parameters such as:

$$
C_{p}=C e^{\beta\left(p-p_{\text {ref }}\right)}
$$

where $C$ is the count rate, $C_{p}$ the corrected count rate, $p_{\text {ref }}$ the pressure of reference and $\beta$ the pressure correction coefficient. The pair of parameters $\left(p_{\text {ref }}, \beta\right)$ depends on the location, the altitude and the characteristics of the detector. Thus, we investigated a possible dependence of the Cal/NM ratio with $p$ that would indicate a different value of $\beta$ for the calibrator and for the 18NM64. Due to the important difference of humidity between the dry and rainy seasons at Doi Inthanon and during the two configurations, we also studied a possible dependence of the Cal/NM ratio with the atmospheric water vapor, $E_{w}$, determined using the Global Data Assimilation System (GDAS) 

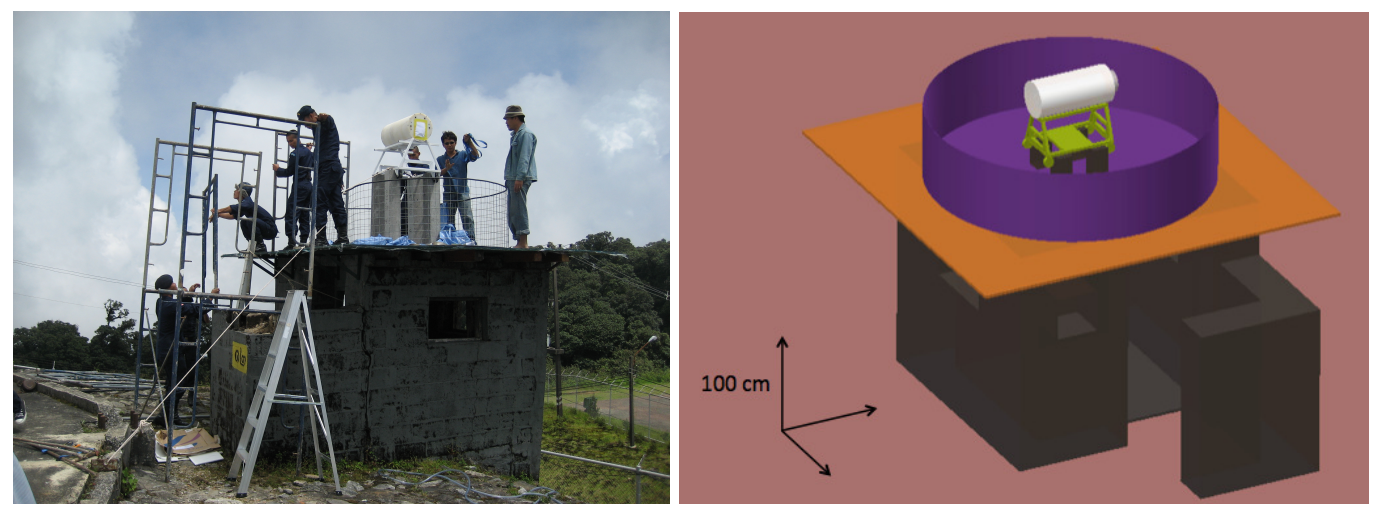

Figure 1: (Left)Installation of the calibrator (white cylinder) outside.(Right) Illustration of the geometry for Monte Carlo simulations of the calibrator outside but nearby the PSNM building [1].

database (http://ready.arl.noaa.gov/gdas1.php). We used the outdoor configuration (providing a larger dataset) to perform a linear regression of the Cal/NM ratio with either $p$ and $E_{w}$. The regression coefficients are respectively $-(2.10 \pm 1.51) \times 10^{-6} \mathrm{mmHg}^{-1}$ and $(1.88 \pm 1.59) \times 10^{-6}$ $\mathrm{mmHg}^{-1}$ and imply an uncertainty of $\mathscr{O}\left(10^{-6}\right)$ in the $\mathrm{Cal} / \mathrm{NM}$ ratio. Therefore we did not apply any correction to the $\mathrm{Cal} / \mathrm{NM}$ ratio. The large datasets taken during the two configurations give a very precise measurement of the $\mathrm{Cal} / \mathrm{NM}$ ratios. The $\mathrm{Cal} / \mathrm{NM}$ ratio inside is higher by $(1.47 \pm 0.03) \times 10^{-4}$.

\section{Monte Carlo simulations}

The computed yield functions of the 18NM64 and of the calibrator at the location of PSNM were determined with a two stage Monte Carlo (MC) simulation using FLUKA [2, 6] as precisely described by [1]. First a set of $3.05 \times 10^{7}$ secondary particles with their properties were collected at the altitude of Doi Inthanon from the simulation of the interaction of protons and alphas in an realistic spherical atmosphere (model based on GDAS and NRLMSISE-00 [17]). The total pressure and the water vapor pressure were respectively $563.4 \mathrm{mmHg}$ and $4.7 \mathrm{mmHg}$, as appropriate for January at Doi Inthanon. The cosmic ray spectrum at the top of the Earth's atmosphere was assumed isotropic and modeled from the local interstellar spectrum described in [18] with a solar "force-field" [7] modulation parameter $\phi=340 \mathrm{MV}$ (mean value during the first six months of 2010). The East-West asymmetry was taken into account by applying the technique of [12] to directly trace each simulated primary particle through the geomagnetic field using the International Geomagnetic Reference Field (IGRF-11). The second stage consisted of simulating the interaction of the secondary particles, chosen within the large population from the first stage, in the detectors and their surroundings. The 18NM64 and calibrator responses in the PSNM station were determined together with the indoor configuration presented in Figure 2 whereas only the response of the calibrator was calculated for the outdoor configuration (Figure 1). The output of the 2 stages was used to extract the simulated count rates of the detectors.

In addition to the precise description of the experimental setups which were used in the FLUKA geometry editor, we implemented, post-MC, an electronic dead time $t_{d}$ in order to es- 


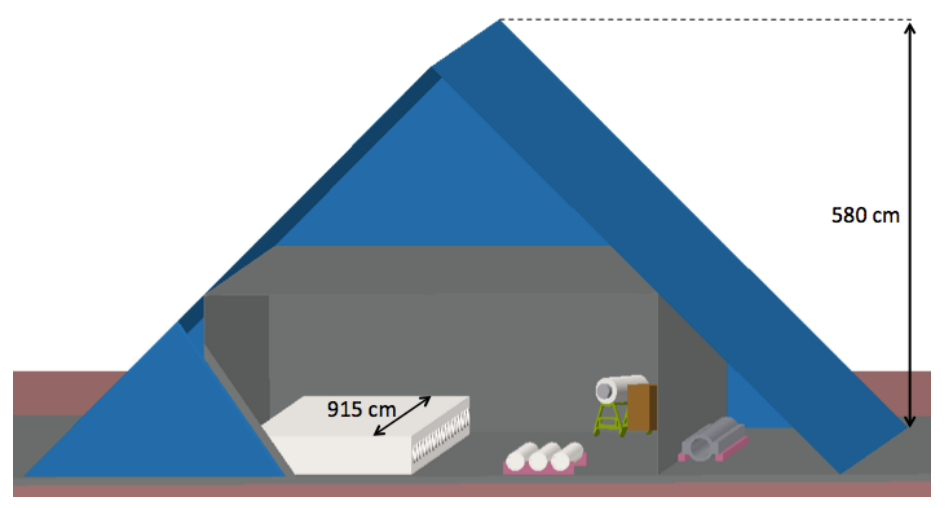

Figure 2: Illustration of the geometry for Monte Carlo simulations of the calibrator inside the PSNM building at Doi Inthanon, Thailand. This cutaway view removes most of the east wall. The calibrator (white cylinder at right) was operated inside the station during June, 2010. The 18-tube NM64 neutron monitor (left) and 3 bare neutron counter tubes (front) have been operating there since 2007. Spare lead rings are kept in a storage room to the right [1].

timate with a better accuracy the efficiency of the detector. We can observe that the count rate of each detector decreases with an increasing dead time (Figure 3). The simulation shows that the dependence of the calibrator is larger than for the 18NM64. This may be explained by the larger efficiency of the neutron's capture via ${ }^{3} \mathrm{He}(n, p)^{3} \mathrm{H}$ than in the boron tubes of the NM64. The sensitivity to the dead time is also found to be dependent on the environment as the decrease is larger for the calibrator outside compared with inside. For our purpose to inter-calibrate several NMs, a precise knowledge of the dead time parameter of both the calibrators and of each NM is crucial to achieve the desired accuracy of $0.2 \%$. For the rest of the simulated data presented here, we used the measured dead times (from 18 to $29 \mu \mathrm{s}$ ) for each of the counters of the 18NM64. In the case of the PH count rate of the calibrator, we used the dead time $(100 \mu \mathrm{s})$ determined from the hourly recorded time delay histograms.

The simulated results are shown in Table 1 . The absolute count rates are overestimated by $13 \%$ and $18 \%$. The choice of the local interstellar spectrum as well as the solar modulation parametrization can introduce uncertainties up to $10 \%$ on the flux of cosmic rays at the top of Earth's atmosphere [8]. Moreover the choices of the hadron interaction model, Monte-Carlo package and atmospheric profile [14] can also contribute to the difference between the simulated and observed count rate. The $\mathrm{Cal} / \mathrm{NM}$ ratios from the $\mathrm{MC}$ agree within a few percent with the observations. A higher $\mathrm{Cal} / \mathrm{NM}$ ratio for the inside configuration is also found in the simulation.

To better understand the differences of sensitivities between the two detectors, we determined using the $\mathrm{MC}$ results the contributions of the main types of secondary particles (proton, neutron, $\mu^{+}, \mu^{-}, \pi^{+}, \pi^{-}, e^{+}, e^{-}$and $\gamma$ ) to the total count rate. The percentages of contributions for each detector and configuration are presented in Table 2. As expected the neutrons and protons are responsible of the majority ( $\sim 95 \%)$ of the counts but significant differences in terms of energy sensitivity can be observed between the 18NM64 and the calibrator and even between the two configurations of the calibrator. For each detector the main contribution comes from neutrons with $10 \mathrm{MeV} \leq E_{k}<1 \mathrm{GeV}$. However, the simulation indicates that the calibrator is much more sensitive 


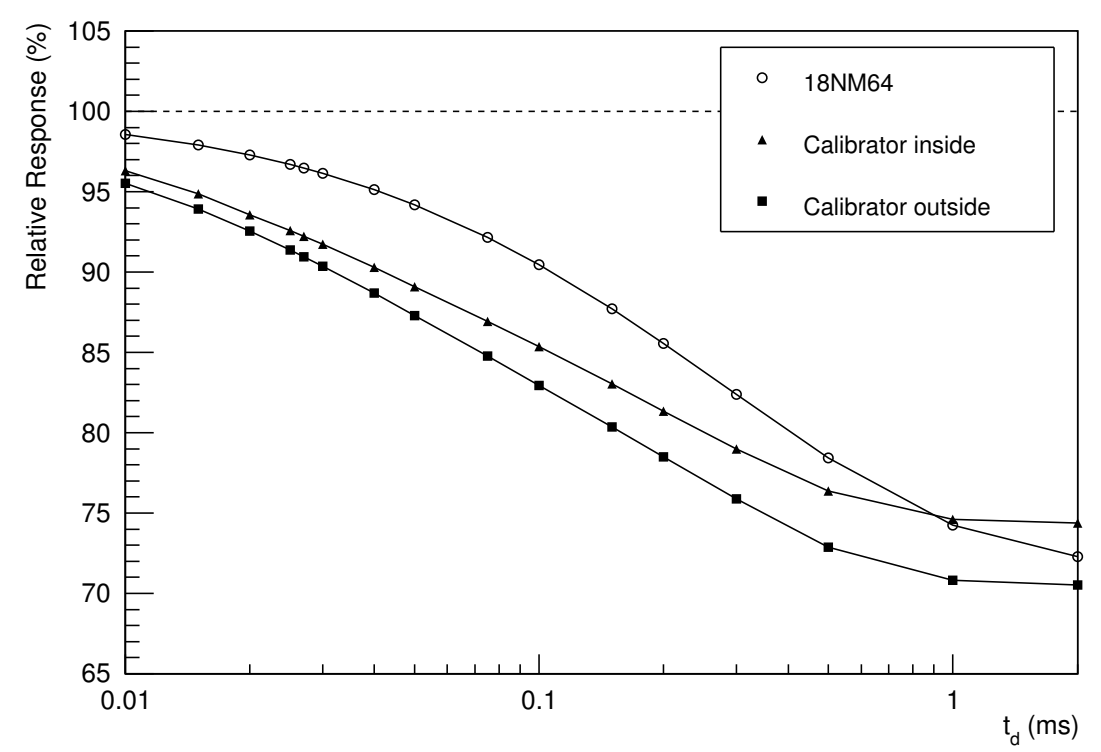

Figure 3: Monte Carlo results for the dependence of count rate on dead time for the 18NM64 and the calibrator inside and outside, relative to zero dead time. Subsequent Monte Carlo results make use of the measured dead times of the 18NM64 tubes (ranging from 18 to $29 \mu \mathrm{s}$ ) and of the calibrator pulse height rates $(100 \mu \mathrm{s})[1]$.

to neutrons with $E_{k}<10 \mathrm{MeV}$ than the 18NM64. The flux of neutrons in that range of energy is more sensitive to the local environment and that can indicate that the calibrator is more sentitive to the conditions of data taking than a NM with a standard NM64 configuration. About the remaining $5 \%$, thus with a lower effect on the total count rate, the calibrator is less (more) sensitive to the hadronic (electromagnetic) components of the secondary particles.

\section{Conclusions}

We operated a calibration neutron monitor together with the Princess Sirindhorn Neutron Monitor (PSNM) located at the top of Doi Inthanon, Thailand. In addition to the calibration of the PSNM count rate with respect to the calibrator count rate and to the worldwide neutron monitor network, the effects of the local environment on the calibration were studied by operating the calibrator inside and outside of the PSNM station. An inter-calibration with an accuracy of $0.2 \%$ proved to be very challenging. Using a detailed MC simulation of the interaction of the cosmic rays in the atmosphere and of the shower of secondary particles in the detectors within their surroundings, we showed that a stable electronic configuration with a known dead time is crucial to achieve that goal. The pool of water helps to standardize the underneath of the calibrator, but the $\mathrm{Cal} / \mathrm{NM}$ ratio depends significantly of the structure of the building where it is located. In order to achieve the desired accuracy, it might be necessary to develop individual MC simulations to study the effects of the surroundings on the sensitivity for each of the NMs independently. Such simulations would also help to estimate a possible dependence of the calibration on solar modulation. 


\begin{tabular}{lccc}
\hline & \multicolumn{2}{c}{ Calibrator } & PSNM \\
\cline { 2 - 3 } & Outside & Inside & \\
\hline$n, E_{k}<10 \mathrm{MeV}$ & $6.5(1)$ & $6.2(2)$ & $1.460(6)$ \\
$n, 10 \mathrm{MeV} \leq E_{k}<1 \mathrm{GeV}$ & $69.2(2)$ & $68.8(4)$ & $63.10(4)$ \\
$n, E_{k} \geq 1 \mathrm{GeV}$ & $8.57(9)$ & $9.3(2)$ & $15.68(3)$ \\
$p$ & $10.5(1)$ & $10.7(2)$ & $15.31(3)$ \\
$\gamma$ & $2.20(3)$ & $1.89(7)$ & $1.213(4)$ \\
$\mu^{-}$ & $1.74(3)$ & $1.81(8)$ & $1.871(6)$ \\
$\mu^{+}$ & $0.25(1)$ & $0.23(2)$ & $0.321(3)$ \\
$\pi^{-}$ & $0.193(9)$ & $0.22(3)$ & $0.333(4)$ \\
$\pi^{+}$ & $0.163(9)$ & $0.16(2)$ & $0.291(4)$ \\
$e^{-}$ & $0.40(1)$ & $0.39(3)$ & $0.221(2)$ \\
$e^{+}$ & $0.38(1)$ & $0.31(3)$ & $0.202(2)$ \\
\hline
\end{tabular}

Table 2: Percentage contributions of various cosmic ray shower components to neutron monitor count rates in Monte Carlo simulations [1].

No atmospheric nor seasonal effects could be clearly determined for the PSNM. Finally, our MC determination of the $\mathrm{Cal} / \mathrm{NM}$ ratio matches the observations within a few percent, including the effect of the building on the calibration. That provides a validation of our model of the PSNM station used in our simulation and our computed yield function.

\section{Acknowledgments}

We thank the Royal Thai Air Force and Chatchai Injai for their kind assistance with the observations, and Andrew Snodin for setting up and maintaining the computing cluster on which our simulations were performed. We also thank André Benadie of North-West University and Leonard Shulman and James Roth of the University of Delaware for their work on the electronics. This research was partially supported by the Thailand Research Fund via Basic Research Grants BRG5180004 and BRG5580001 and Royal Golden Jubilee fellowship PHD/0136/2552. P.-S. M. was partially supported under the postdoctoral research sponsorship of Mahidol University. This work was also financially supported by the South African National Antarctic Programme of the National Research Foundation, and by the United States National Science Foundation via awards PLR-1341562, PLR-1245939 and their predecessors.

\section{References}

[1] N. Aiemsa-ad et al., Measurement and simulation of neutron monitor count rate dependence on surrounding structure, J. Geophys. Res. Space Physics, 120, doi: 10.1002/2015JA021249 (2015).

[2] T. T. Bohlen, F. Cerutti, M. P. W. Chin, A. Fassò, A. Ferrari, P. G. Ortega, A. Mairani, P. R. Sala, G. Smirnov, and V. Vlachoudis, The FLUKA Code: developments and challenges for high energy and medical applications, Nuclear Data Sheets, 120, 211-214 (2014). 
[3] R. A. Caballero-Lopez and H. Moraal, Cosmic-ray yield and response functions in the atmosphere, J. Geophys. Res., 117, A12103, doi:10.1029/2012JA011794 (2012).

[4] H. Carmichael, IQSY Instruction Manual No. 7, London: IQSY Secreatariat, (1964).

[5] J. Clem and L. Dorman, Neutron monitor response functions, Space Sci. Rev., 93, 335 (2000).

[6] A. Ferrari, P. R. Sala, A. Fassò and J. Ranft, FLUKA: a multi-particle transport code, CERN-2005-10, INFN/TC_05/11, SLAC-R-773 (2005).

[7] L. J. Gleeson and W. I. Axford, 1968, Solar modulation of Galactic cosmic rays, Astrophys. J., 154, 1011, (1968).

[8] K. Herbst et al., On the importance of the local interstellar spectrum for the solar modulation parameter, J. Geophys. Res., 115, D00I20, doi:10.1029/2009JD012557 (2010).

[9] H. Krüger, H. Moraal, J. W. Bieber, J. M. Clem, P. A. Evenson, K. R. Pyle, M. L. Duldig, and J. E. Humble, First Results of a Mobile Neutron Monitor to Intercalibrate the Worldwide Network, 28th International Cosmic Ray Conference, IUPAP, Tsukuba, Japan, vol. 6, 3441-3444 (2003).

[10] H. Krüger, H. Moraal, J. W. Bieber, J. M. Clem, P. A. Evenson, K. R. Pyle, M. L. Duldig, and J. E. Humble, A calibration neutron monitor: Energy response and instrumental temperature sensitivity, J. Geophys. Res., 113, A08101, doi:10.1029/2008JA013229 (2008).

[11] H. Krüger \& H. Moraal, A calibration neutron monitor: Statistical accuracy and environmental sensitivity, Adv. Space Res., 46, 1394-1399, doi:10.1016/j.asr.2010.07.008 (2010).

[12] Z. Lin, J. W. Bieber, and P. Evenson, Electron trajectories in a model magnetosphere: Simulation and observation under active conditions, J. Geophys. Res., 100 , 23543-23550, doi:10.1029/95JA02696 (1995).

[13] A. L. Mishev, I. G. Usoskin and G. A. Kovaltsov, Neutron monitor yield function: New improved computations, J. Geophys. Res., 118, 2783-2788, doi:10.1002/jgra.50325 (2013).

[14] A. L. Mishev and P. I. Y. Velinov, Influence of hadron and atmospheric models on computation of cosmic ray ionization in the atmosphere - Extension to heavy nuclei, J. Atm. Solar-Terr. Phys., 120, 111-120, doi:10.1016/j.jastp.2014.09.007 (2014).

[15] H. Moraal, A. Belov, and J. M. Clem, Design and co-ordination ofmulti-station international neutron monitor networks, Space Sci. Rev., 93, 283-303 (200).

[16] H. Moraal, H. Krüger, A. Benadie, and D. de Villiers, Calibration of the Sanae and Hermanus Neutron Monitors, 28th International Cosmic Ray Conference, IUPAP, Tsukuba, Japan, vol. 6, 3453-3456 (2003).

[17] J. M. Picone, A. E. Hedin, D. P. Drob, A. C. Aikin, NRLMSISE-00 empirical model of the atmosphere: Statistical comparisons and scientific issues, J. Geophys. Res., 107, 1468, doi:10.1029/2002JA009430 (2002).

[18] I. G. Usoskin, G. A. Bazilevskaya and G. A. Kovaltsov, Solar modulation parameter for cosmic rays since 1936 reconstructed from ground-based neutron monitors and ionization, J. Geophys. Res., 116, A02104, doi:10.1029/2010JA016105 (2011). 